\title{
Copper(I) Complexes with Bis(trimethylsilyl)acetylene: Role of Ancillary Ligands in Determining $\pi$ Backbonding Interactions
}

\author{
Jeffery S. Thompson, Alexander Z. Bradley, Kyung-Ho Park, Kerwin D. Dobbs, Will \\ Marshall \\ Central Research and Development Department \\ DuPont Company \\ Wilmington, DE 19880-0328
}

Preparation of 3. All manipulations were performed in a dry box under nitrogen atmosphere. Cuprous chloride $(1.5 \mathrm{~g}, 15.153 \mathrm{mmol})$ was mixed with BTMSA $(2.582 \mathrm{~g}$, $15.153 \mathrm{mmol})$ in ether $(30 \mathrm{~mL})$. The resultant mixture was stirred at room temperature for $0.5 \mathrm{~h}$. At the same time 2,2,7-trimethyloctane-3,5-dione (H(tod), $2.792 \mathrm{~g}, 15.153 \mathrm{mmol}$ ) was mixed with $\mathrm{NaH}(60 \%, 0.606 \mathrm{~g}, 15.153 \mathrm{mmol})$ in ether $(30 \mathrm{~mL})$; this mixture was also stirred at room temperature for $0.5 \mathrm{~h}$. The latter mixture was added to the former mixture, and the reaction mixture was stirred at room temperature overnight. The solvent was removed under reduced pressure. The resultant residue was treated with hexane (40 $\mathrm{mL}$ ), and filtered. The filtrate was concentrated under reduced pressure to afford $6.02 \mathrm{~g}$ of crude product (95\%) as a white solid. This solid was recrystallized in hexane at $-30{ }^{\circ} \mathrm{C}$ to give $3 \mathrm{~g}$ of pure product. ${ }^{\mathrm{I}} \mathrm{H} \mathrm{NMR}\left(300 \mathrm{MHz}, \mathrm{CD}_{2} \mathrm{Cl}_{2}\right) \delta 5.45(\mathrm{~s}, 1 \mathrm{H}), 2.09-2.04(\mathrm{~m}$, $3 \mathrm{H}), 1.13(\mathrm{~s}, 9 \mathrm{H}), 0.92(\mathrm{~d}, J=6.2 \mathrm{~Hz}, 6 \mathrm{H}), 0.31(\mathrm{~s}, 18 \mathrm{H}) ;{ }^{13} \mathrm{C} \mathrm{NMR}\left(75 \mathrm{MHz}, \mathrm{CD}_{2} \mathrm{Cl}_{2}\right): \delta$ 199.4, 193.9, 114.6, 94.9, 51.8, 41.4, 28.9, 27.0, 23.1, -0.01.

Preparation of 4. All manipulations were performed in a dry box under nitrogen atmosphere. A 100-mL round-bottom flask was charged with $[\mathrm{Cu}(\mathrm{CH} 3 \mathrm{CN}) 4] \mathrm{SO} 3 \mathrm{CF} 3$ $(1.0 \mathrm{~g}, 2.6 \mathrm{mmole})$, bistrimethylsilylacetylene ( $1.5 \mathrm{~g}, 8.8 \mathrm{mmole})$, and diethyl ether $(20 \mathrm{~mL})$. In a separate $100-\mathrm{mL}$ round-bottom flask, $1.5 \mathrm{M}$ t-butyl lithium $(1.7 \mathrm{~mL}, 2.6$ mmole) was added to a solution of $\mathrm{N}, \mathrm{N}^{\prime}$-diisobutyl-2,4-pentanediketimine $(0.550 \mathrm{~g}, 2.6$ mmole). After $0.5 \mathrm{~h}$, the solutions were combined. The combined solution changed from a cloudy white suspension to a dark-brown solution after the uptake of all solids. After $2 \mathrm{~h}$, the solution was concentrated to a solid/sludge, extracted with pentane $(3 \times 15 \mathrm{~mL})$, filtered and concentrated to give a brown solid $(0.680 \mathrm{~g}, 59 \%$ yield $)$. A single crystal suitable for xray analysis was grown from a pentane solution at $-32^{\circ} \mathrm{C}$. ${ }^{1} \mathrm{HNMR} \delta\left(\mathrm{C}_{6} \mathrm{D}_{6}\right)$ $0.26(\mathrm{~s}, 18 \mathrm{H}), 0.99(\mathrm{~d}, 16 \mathrm{H}, \mathrm{J}=6.77 \mathrm{~Hz}), 1.83(\mathrm{~m}, 2 \mathrm{H}) 1.94(\mathrm{~s}, 6 \mathrm{H}), 3.46(\mathrm{~d}, 4 \mathrm{H}, \mathrm{J}=6.93$ $\mathrm{Hz}), 4.57(\mathrm{~s}, 1 \mathrm{H}) .{ }^{13} \mathrm{CNMR} \delta\left(\mathrm{C}_{6} \mathrm{D}_{6}\right) \quad 162.25,125.29,96.17,62.12,32.07,20.99,19.89$, 0.92 .

Reference 16.

Frisch, M. J.; Trucks, G. W.; Schlegel, H. B.; Scuseria, G. E.; Robb, M. A.; Cheeseman, J. R.; Montgomery, J. A., Jr.; Vreven, T.; Kudin, K. N.; Burant, J. C.; Millam, J. M.; Iyengar, S. S.; Tomasi, J.; Barone, V.; Mennucci, B.; Cossi, M.; Scalmani, G.; Rega, N.; Petersson, G. A.; Nakatsuji, H.; Hada, M.; Ehara, M.; Toyota, K.; Fukuda, R.; Hasegawa, J.; Ishida, M.; Nakajima, T.; Honda, Y.; Kitao, O.; Nakai, H.; Klene, M.; Li, X.; Knox, J. E.; Hratchian, H. P.; Cross, J. B.; Adamo, C.; Jaramillo, J.; Gomperts, R.; Stratmann, R. 
E.; Yazyev, O.; Austin, A. J.; Cammi, R.; Pomelli, C.; Ochterski, J. W.; Ayala, P. Y.; Morokuma, K.; Voth, G. A.; Salvador, P.; Dannenberg, J. J.; Zakrzewski, V. G.; Dapprich, S.; Daniels, A. D.; Strain, M. C.; Farkas, O.; Malick, D. K.; Rabuck, A. D.; Raghavachari, K.; Foresman, J. B.; Ortiz, J. V.; Cui, Q.; Baboul, A. G.; Clifford, S.; Cioslowski, J.; Stefanov, B. B.; Liu, G.; Liashenko, A.; Piskorz, P.; Komaromi, I.; Martin, R. L.; Fox, D. J.; Keith, T.; Al-Laham, M. A.; Peng, C. Y.; Nanayakkara, A.; Challacombe, M.; Gill, P. M. W.; Johnson, B.; Chen, W.; Wong, M. W.; Gonzalez, C.; Pople, J. A. Gaussian 03, revision B.04; Gaussian, Inc.: Pittsburgh, PA, 2003.

Table of Crystallographic Parameters.

\begin{tabular}{|c|c|c|}
\hline & 3 & 4 \\
\hline empirical formula & $\mathrm{C}_{19} \mathrm{H}_{37} \mathrm{CuO}_{2} \mathrm{Si}_{2}$ & $\mathrm{C}_{21} \mathrm{H}_{43} \mathrm{CuN}_{2} \mathrm{Si}_{2}$ \\
\hline FW & 417.21 & 443.29 \\
\hline cryst.color, form & $\begin{array}{l}\text { colorless, } \\
\text { irreg.block }\end{array}$ & $\begin{array}{l}\text { Colorless, } \\
\text { irreg. block }\end{array}$ \\
\hline cryst system & monoclinic & Triclinic \\
\hline Space group & $\mathrm{P} 2(1) / \mathrm{c}$ & $P-1$ \\
\hline $\mathrm{a}(\AA)$ & $11.7663(15)$ & $11.502(2)$ \\
\hline $\mathrm{b}(\AA)$ & $20.368(2)$ & $17.021(3)$ \\
\hline$c(\AA)$ & $11.1494(14)$ & $6.9570(14)$ \\
\hline$\alpha(\operatorname{deg})$ & 90 & $96.20(3)$ \\
\hline$\beta(\operatorname{deg})$ & $117.044(3)$ & $103.00(3)$ \\
\hline$\gamma(\operatorname{deg})$ & 90 & $89.58(3)$ \\
\hline $\mathrm{V}\left(\AA^{3}\right)$ & $2379.8(5)$ & $1319.2(5)$ \\
\hline $\mathrm{Z}$ & 4 & 2 \\
\hline $\operatorname{density}(\mathrm{g} / \mathrm{cm} 3)$ & 1.164 & 1.116 \\
\hline abs. $\mu\left(\mathrm{mm}^{-1}\right)$ & 1.026 & 0.926 \\
\hline $\mathrm{F}(000)$ & 896 & 480 \\
\hline cryst size(mm) & $0.16 \times 0.05 \times 0.03$ & $0.30 \times 0.30 \times 0.10$ \\
\hline Temp(degrees C) & -100 & -100 \\
\hline scan mode & $\omega$ & $\Omega$ \\
\hline detector & Bruker-CCD & Bruker-CCD \\
\hline$\theta_{\max }(\mathrm{deg})$ & 28.3 & 24.1 \\
\hline no. obsrvd. refs & 10985 & 7087 \\
\hline no. uniq. Refs & 5451 & 3717 \\
\hline $\mathrm{R}_{\text {merge }}$ & 0.085 & 0.052 \\
\hline no. params & 228 & 248 \\
\hline$S^{* *}$ & 1.014 & 0.955 \\
\hline $\operatorname{Rindices}[\mathrm{I}>2 \sigma(\mathrm{I})]^{*}$ & $w R 2=0.138, R 1=0.077$ & $w R 2=0.088, R 1=0.036$ \\
\hline $\mathrm{R}$ indices (all data) ${ }^{*}$ & $w R 2=0.180, R 1=0.187$ & $w R 2=0.091, R 1=0.045$ \\
\hline max diff peak, hole $\left(\mathrm{e} / \AA^{3}\right)$ & $0.980,-1.137$ & $0.343,-0.477$ \\
\hline
\end{tabular}


$* R 1=\Sigma|| F_{\mathrm{o}}|-| F_{\mathrm{c}}|| / \Sigma\left|F_{\mathrm{o}}\right|, w R 2=\left\{\Sigma\left[w\left(F_{\mathrm{o}}^{2}-F_{\mathrm{c}}^{2}\right)^{2}\right] / \Sigma\left[w\left(F_{\mathrm{o}}^{2}\right)^{2}\right]\right\}^{1 / 2}$ (sometimes denoted as $\left.R_{\mathrm{w}} 2\right)$

$* *$ GooF $=S=\left\{\Sigma\left[w\left(F_{\mathrm{o}}^{2}-F_{\mathrm{c}}^{2}\right)^{2}\right] /(n-p)\right\}^{1 / 2}$, where $n$ is the number of reflections, and $p$ is the total number of refined parameters.

\section{$\underline{\text { X-RAY CRYSTAL STRUCTURE ANALYSIS Compound } 3}$}

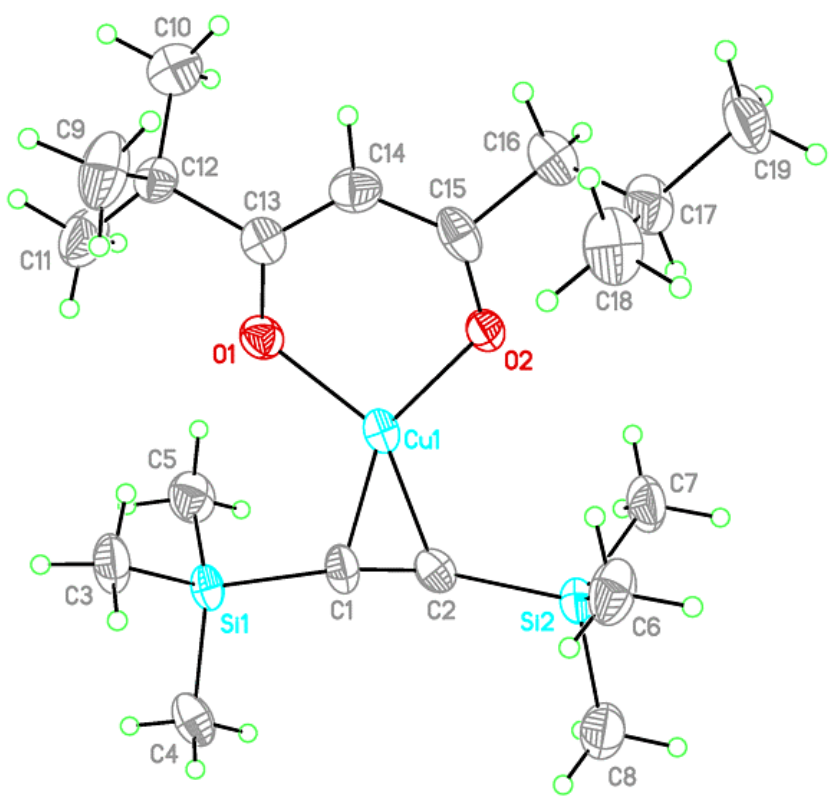

CRYSTAL DATA: C19 H37 Cu O2 Si2, from diethylether, colorless, irregular block, $\sim 0.160 \times 0.050 \times 0.030 \mathrm{~mm}$, monoclinic , P21/c, a = 11.7663(15) $\AA, \mathrm{b}=20.368(2) \AA, \mathrm{c}=$ 11.1494(14) $\AA$, beta $=117.044(3)^{\circ}, \mathrm{Vol}=2379.8(5) \AA^{3}, \mathrm{Z}=4, \mathrm{~T}=-100 .{ }^{\circ} \mathrm{C}$, Formula weight $=417.21$, Density $=1.164 \mathrm{~g} / \mathrm{cm}^{3}, \mu(\mathrm{Mo})=1.03 \mathrm{~mm}^{-1}$

DATA COLLECTION: Bruker SMART 1K CCD system, MoKalpha radiation, standard focus tube , anode power $=50 \mathrm{kV}$ x $40 \mathrm{~mA}$, crystal to plate distance $=4.9 \mathrm{~mm}$, $512 \times 512$ pixels/frame, hemisphere data aquisition, total scans $=4$, total frames $=2660$, oscillation $/$ frame $=-0.15^{\circ}$, exposure $/$ frame $=30.0 \mathrm{sec} /$ frame, maximum detector swing angle $=-28.0^{\circ}$, beam center $=(255.25,253.13)$, in plane spot width $=0.00$, omega half width $=0.00$, SAINT integration, hkl $\mathrm{min} / \max =(-12,15,-25,26,-14,8)$, data input to shelx $=10985$, unique data $=5451$, two-theta range $=3.88$ to $56.60^{\circ}$, completeness to two-theta $56.60=92.30 \%, \mathrm{R}($ int-xl $)=0.0845$, SADABS correction applied. 
SOLUTION AND REFINEMENT: Structure solved using XS(Shelxtl), refined using shelxtl software package, refinement by full-matrix least squares on $\mathrm{F}^{2}$, scattering factors from Int. Tab. Vol C Tables 4.2.6.8 and 6.1.1.4, number of data $=5451$, number of restraints $=0$, number of parameters $=228$, data/parameter ratio $=23.91$, goodness-of-fit on $\mathrm{F}^{2}=1.01, \mathrm{R}$ indices[I>4sigma(I)] $\mathrm{R} 1=0.0771, \mathrm{wR} 2=0.1379, \mathrm{R}$ indices(all data) $\mathrm{R} 1$ $=0.1865, \mathrm{wR} 2=0.1798$, max difference peak and hole $=0.980$ and $-1.137 \mathrm{e} / \AA^{3}$, All hydrogen atoms have been idealized as riding hydrogens. The rotation of the methyl groups are refined.

RESULTS: This study determines the structure of Bis(trimethylsilyl)acetylene(2,2,7trimethyloctane-3,5-dionato)copper(I) for advanced interconnect materials research(APEX 480). The asymmetric unit contains one molecule as shown in figure 1 with thermal ellipsoids drawn to the $50 \%$ probability level. The predicted structure is confirmed. An earlier crystallization from hexane yielded a poor disordered structure that was solved from synchrotron data as 02349 .

Table 1. Atomic coordinates ( $x$ 10^4) and equivalent isotropic displacement parameters $\left(A^{\wedge} 2 \times 10^{\wedge} 3\right)$ for 02388 .

$U(e q)$ is defined as one third of the trace of the orthogonalized Uij tensor.

\begin{tabular}{|c|c|c|c|c|}
\hline & $\mathbf{x}$ & $y$ & $\mathbf{z}$ & $\mathrm{U}(\mathrm{eq})$ \\
\hline $\mathrm{Cu}(1)$ & $2190(1)$ & $6288(1)$ & $6638(1)$ & $27(1)$ \\
\hline Si (1) & $4784(2)$ & $5383(1)$ & $7641(2)$ & $29(1)$ \\
\hline$O(1)$ & $1733(4)$ & $5800(2)$ & $4998(4)$ & $32(1)$ \\
\hline$C(1)$ & $3875(6)$ & $6019(3)$ & $8064(7)$ & $31(2)$ \\
\hline $\mathrm{Si}(2)$ & $3093(2)$ & $7073(1)$ & $9522(2)$ & $31(1)$ \\
\hline$O(2)$ & $678(4)$ & $6820(2)$ & $5978(4)$ & $31(1)$ \\
\hline$C(2)$ & $3456(5)$ & $6453(3)$ & $8515(6)$ & $28(2)$ \\
\hline$C(3)$ & $3789(7)$ & $4628(3)$ & $7050(7)$ & $46(2)$ \\
\hline$C(4)$ & $6268(6)$ & $5187(4)$ & $9190(7)$ & $42(2)$ \\
\hline$C(5)$ & $5103(7)$ & $5740(4)$ & $6305(7)$ & $46(2)$ \\
\hline$C(6)$ & $1641(6)$ & $6787(4)$ & $9613(8)$ & $48(2)$ \\
\hline$C(7)$ & $2810(8)$ & $7861(3)$ & $8620(7)$ & $52(2)$ \\
\hline$C(8)$ & $4458(7)$ & $7139(4)$ & $11205(7)$ & $51(2)$ \\
\hline$C(9)$ & $-395(7)$ & $4845(4)$ & $2757(9)$ & $60(2)$ \\
\hline$C(10)$ & $-145(8)$ & $5772(4)$ & $1422(7)$ & $60(2)$ \\
\hline$C(11)$ & $1720(7)$ & $5122(4)$ & $2935(8)$ & $54(2)$ \\
\hline$C(12)$ & $468(6)$ & $5397(3)$ & $2759(6)$ & $31(2)$ \\
\hline$C(13)$ & $675(6)$ & $5858(3)$ & $3941(7)$ & $29(2)$ \\
\hline$C(14)$ & $-286(6)$ & $6290(4)$ & $3833(7)$ & $37(2)$ \\
\hline$C(15)$ & $-225(6)$ & $6742(3)$ & $4801(8)$ & $35(2)$ \\
\hline$C(16)$ & $-1341(6)$ & $7207(3)$ & $4397(7)$ & $42(2)$ \\
\hline$C(17)$ & $-1740(6)$ & $7378(4)$ & $5459(8)$ & $43(2)$ \\
\hline$C(18)$ & $-2160(7)$ & $6775(4)$ & $5960(9)$ & $57(2)$ \\
\hline$C(19)$ & $-2778(7)$ & $7906(4)$ & $4947(9)$ & $64(3)$ \\
\hline
\end{tabular}


Table 2. Bond lengths [A] and angles [deg] for 02388 .

\begin{tabular}{|c|c|}
\hline $\mathrm{Cu}(1)-\mathrm{O}(2)$ & $1.920(4)$ \\
\hline $\mathrm{Cu}(1)-\mathrm{O}(1)$ & $1.931(4)$ \\
\hline $\mathrm{Cu}(1)-\mathrm{C}(2)$ & $1.969(6)$ \\
\hline $\mathrm{Cu}(1)-\mathrm{C}(1)$ & $1.974(7)$ \\
\hline $\mathrm{Si}(1)-\mathrm{C}(5)$ & $1.843(7)$ \\
\hline $\operatorname{Si}(1)-C(4)$ & $1.857(7)$ \\
\hline $\operatorname{Si}(1)-C(3)$ & $1.862(7)$ \\
\hline $\mathrm{Si}(1)-\mathrm{C}(1)$ & $1.871(6)$ \\
\hline$O(1)-C(13)$ & $1.272(7)$ \\
\hline$C(1)-C(2)$ & $1.225(8)$ \\
\hline $\operatorname{Si}(2)-C(8)$ & $1.838(7)$ \\
\hline $\operatorname{Si}(2)-C(7)$ & $1.842(7)$ \\
\hline $\operatorname{Si}(2)-C(6)$ & $1.852(7)$ \\
\hline $\operatorname{Si}(2)-C(2)$ & $1.864(7)$ \\
\hline$O(2)-C(15)$ & $1.268(8)$ \\
\hline$C(9)-C(12)$ & $1.514(9)$ \\
\hline$C(10)-C(12)$ & $1.533(10)$ \\
\hline$C(11)-C(12)$ & $1.503(9)$ \\
\hline$C(12)-C(13)$ & $1.546(9)$ \\
\hline$C(13)-C(14)$ & $1.394(9)$ \\
\hline$C(14)-C(15)$ & $1.395(9)$ \\
\hline$C(15)-C(16)$ & $1.514(9)$ \\
\hline$C(16)-C(17)$ & $1.500(9)$ \\
\hline$C(17)-C(18)$ & $1.521(10)$ \\
\hline$C(17)-C(19)$ & $1.529(9)$ \\
\hline $\mathrm{O}(2)-\mathrm{Cu}(1)-\mathrm{O}(1)$ & $96.75(18)$ \\
\hline $\mathrm{O}(2)-\mathrm{Cu}(1)-\mathrm{C}(2)$ & $115.7(2)$ \\
\hline $\mathrm{O}(1)-\mathrm{Cu}(1)-\mathrm{C}(2)$ & $147.6(2)$ \\
\hline $\mathrm{O}(2)-\mathrm{Cu}(1)-\mathrm{C}(1)$ & $151.9(2)$ \\
\hline $\mathrm{O}(1)-\mathrm{Cu}(1)-\mathrm{C}(1)$ & $111.4(2)$ \\
\hline $\mathrm{C}(2)-\mathrm{Cu}(1)-\mathrm{C}(1)$ & $36.2(2)$ \\
\hline$C(5)-S i(1)-C(4)$ & $112.6(3)$ \\
\hline$C(5)-S i(1)-C(3)$ & $111.5(3)$ \\
\hline$C(4)-S i(1)-C(3)$ & $109.0(3)$ \\
\hline$C(5)-S i(1)-C(1)$ & $106.3(3)$ \\
\hline$C(4)-S i(1)-C(1)$ & $108.5(3)$ \\
\hline$C(3)-S i(1)-C(1)$ & $108.8(3)$ \\
\hline $\mathrm{C}(13)-\mathrm{O}(1)-\mathrm{Cu}(1)$ & $124.2(4)$ \\
\hline$C(2)-C(1)-S i(1)$ & $170.0(6)$ \\
\hline $\mathrm{C}(2)-\mathrm{C}(1)-\mathrm{Cu}(1)$ & $71.7(4)$ \\
\hline $\mathrm{Si}(1)-\mathrm{C}(1)-\mathrm{Cu}(1)$ & $118.0(3)$ \\
\hline$C(8)-S i(2)-C(7)$ & $110.5(4)$ \\
\hline$C(8)-S i(2)-C(6)$ & $111.5(4)$ \\
\hline$C(7)-S i(2)-C(6)$ & $111.2(4)$ \\
\hline$C(8)-S i(2)-C(2)$ & $109.3(3)$ \\
\hline$C(7)-S i(2)-C(2)$ & $107.2(3)$ \\
\hline$C(6)-S i(2)-C(2)$ & $106.8(3)$ \\
\hline$C(15)-O(2)-C u(1)$ & $121.3(4)$ \\
\hline$C(1)-C(2)-S i(2)$ & $168.8(6)$ \\
\hline$C(1)-C(2)-C u(1)$ & $72.1(5)$ \\
\hline $\mathrm{Si}(2)-\mathrm{C}(2)-\mathrm{Cu}(1)$ & $119.0(3)$ \\
\hline$C(11)-C(12)-C(9)$ & $110.0(6)$ \\
\hline$C(11)-C(12)-C(10)$ & $108.2(6)$ \\
\hline
\end{tabular}




$\begin{array}{ll}C(9)-C(12)-C(10) & 110.5(6) \\ C(11)-C(12)-C(13) & 110.5(5) \\ C(9)-C(12)-C(13) & 107.3(5) \\ C(10)-C(12)-C(13) & 110.4(6) \\ O(1)-C(13)-C(14) & 123.6(6) \\ O(1)-C(13)-C(12) & 116.2(5) \\ C(14)-C(13)-C(12) & 120.2(6) \\ C(13)-C(14)-C(15) & 126.5(6) \\ O(2)-C(15)-C(14) & 127.5(6) \\ O(2)-C(15)-C(16) & 115.7(6) \\ C(14)-C(15)-C(16) & 116.8(6) \\ C(17)-C(16)-C(15) & 116.9(6) \\ C(16)-C(17)-C(18) & 111.9(6) \\ C(16)-C(17)-C(19) & 110.6(6) \\ C(18)-C(17)-C(19) & 111.6(6)\end{array}$

Symmetry transformations used to generate equivalent atoms:

Table 3. Anisotropic displacement parameters $\left(A^{\wedge} 2 \times 10^{\wedge} 3\right)$ for 02388 . The anisotropic displacement factor exponent takes the form: $-2 \mathrm{pi}^{\wedge} 2\left[\mathrm{~h}^{\wedge} 2 \mathrm{a}^{{ }^{\wedge} 2} \mathrm{U} 11+\ldots+2 \mathrm{~h} \mathrm{k} \mathrm{a}^{*} \mathrm{~b} * \mathrm{U} 12\right]$

\begin{tabular}{lllrrrr}
\hline & & & & & & \\
& U11 & U22 & U33 & U23 & U13 & U12 \\
& & & & & & \\
\hline $\mathrm{Cu}(1)$ & $28(1)$ & $22(1)$ & $34(1)$ & $1(1)$ & $16(1)$ & $3(1)$ \\
$\mathrm{Si}(1)$ & $30(1)$ & $25(1)$ & $38(1)$ & $-2(1)$ & $20(1)$ & $6(1)$ \\
O(1) & $28(2)$ & $34(3)$ & $34(3)$ & $2(2)$ & $14(2)$ & $4(2)$ \\
$\mathrm{C}(1)$ & $45(4)$ & $20(3)$ & $41(4)$ & $2(3)$ & $30(4)$ & $4(3)$ \\
$\mathrm{Si}(2)$ & $35(1)$ & $27(1)$ & $34(1)$ & $-2(1)$ & $18(1)$ & $6(1)$ \\
O(2) & $28(2)$ & $28(3)$ & $32(3)$ & $-4(2)$ & $8(2)$ & $6(2)$ \\
$\mathrm{C}(2)$ & $22(3)$ & $28(4)$ & $37(4)$ & $3(3)$ & $15(3)$ & $1(3)$ \\
$\mathrm{C}(3)$ & $61(5)$ & $25(4)$ & $57(5)$ & $-6(4)$ & $33(4)$ & $4(4)$ \\
$\mathrm{C}(4)$ & $35(4)$ & $35(4)$ & $59(5)$ & $4(4)$ & $24(4)$ & $12(3)$ \\
$\mathrm{C}(5)$ & $43(4)$ & $50(5)$ & $51(5)$ & $1(4)$ & $28(4)$ & $8(4)$ \\
$\mathrm{C}(6)$ & $37(4)$ & $59(5)$ & $58(5)$ & $-20(4)$ & $30(4)$ & $-4(4)$ \\
$\mathrm{C}(7)$ & $82(6)$ & $28(4)$ & $55(5)$ & $3(4)$ & $40(5)$ & $15(4)$ \\
$\mathrm{C}(8)$ & $52(5)$ & $49(5)$ & $50(5)$ & $-13(4)$ & $21(4)$ & $6(4)$ \\
$\mathrm{C}(9)$ & $65(5)$ & $47(5)$ & $79(6)$ & $-28(5)$ & $42(5)$ & $-20(4)$ \\
$\mathrm{C}(10)$ & $66(5)$ & $62(6)$ & $43(5)$ & $-4(4)$ & $16(4)$ & $12(5)$ \\
$\mathrm{C}(11)$ & $46(4)$ & $68(6)$ & $51(5)$ & $-21(4)$ & $25(4)$ & $2(4)$ \\
$\mathrm{C}(12)$ & $26(3)$ & $33(4)$ & $34(4)$ & $-5(3)$ & $13(3)$ & $-5(3)$ \\
$\mathrm{C}(13)$ & $29(3)$ & $27(4)$ & $37(4)$ & $3(3)$ & $19(3)$ & $-4(3)$ \\
$\mathrm{C}(14)$ & $34(3)$ & $39(4)$ & $34(4)$ & $1(4)$ & $12(3)$ & $-4(4)$ \\
$\mathrm{C}(15)$ & $38(4)$ & $23(4)$ & $54(5)$ & $13(4)$ & $30(4)$ & $7(3)$ \\
$\mathrm{C}(16)$ & $40(4)$ & $34(4)$ & $52(5)$ & $6(4)$ & $22(4)$ & $6(4)$ \\
$\mathrm{C}(17)$ & $26(3)$ & $49(5)$ & $57(5)$ & $-9(4)$ & $21(3)$ & $1(4)$ \\
$\mathrm{C}(18)$ & $57(5)$ & $49(5)$ & $79(6)$ & $-4(5)$ & $44(5)$ & $-5(4)$ \\
$\mathrm{C}(19)$ & $37(4)$ & $56(6)$ & $86(7)$ & $-23(5)$ & $16(4)$ & $14(4)$ \\
& & & & & &
\end{tabular}


Table 4. Hydrogen coordinates $\left(x^{10 \wedge} 4\right)$ and isotropic displacement parameters $\left(A^{\wedge} 2 \times 10^{\wedge} 3\right)$ for 02388 .

\begin{tabular}{|c|c|c|c|c|}
\hline & $\mathbf{x}$ & $y$ & $\mathbf{z}$ & $\mathrm{U}$ (eq) \\
\hline $\mathrm{H}(3 \mathrm{~A})$ & 2948 & 4742 & 6323 & 68 \\
\hline $\mathrm{H}(3 \mathrm{~B})$ & 3688 & 4433 & 7799 & 68 \\
\hline $\mathrm{H}(3 \mathrm{C})$ & 4207 & 4312 & 6717 & 68 \\
\hline $\mathrm{H}(4 \mathrm{~A})$ & 6814 & 4905 & 8958 & 63 \\
\hline $\mathrm{H}(4 \mathrm{~B})$ & 6050 & 4958 & 9831 & 63 \\
\hline $\mathrm{H}(4 \mathrm{C})$ & 6723 & 5595 & 9597 & 63 \\
\hline $\mathrm{H}(5 \mathrm{~A})$ & 4293 & 5830 & 5510 & 68 \\
\hline $\mathrm{H}(5 \mathrm{~B})$ & 5604 & 5430 & 6067 & 68 \\
\hline $\mathrm{H}(5 \mathrm{C})$ & 5583 & 6150 & 6629 & 68 \\
\hline $\mathrm{H}(6 \mathrm{~A})$ & 940 & 6743 & 8700 & 72 \\
\hline $\mathrm{H}(6 \mathrm{~B})$ & 1405 & 7105 & 10120 & 72 \\
\hline $\mathrm{H}(6 \mathrm{C})$ & 1809 & 6360 & 10067 & 72 \\
\hline $\mathrm{H}(7 \mathrm{~A})$ & 2079 & 7817 & 7727 & 78 \\
\hline $\mathrm{H}(7 \mathrm{~B})$ & 3570 & 7983 & 8523 & 78 \\
\hline $\mathrm{H}(7 \mathrm{C})$ & 2631 & 8202 & 9132 & 78 \\
\hline $\mathrm{H}(8 \mathrm{~A})$ & 5218 & 7271 & 11119 & 77 \\
\hline $\mathrm{H}(8 \mathrm{~B})$ & 4610 & 6713 & 11660 & 77 \\
\hline $\mathrm{H}(8 \mathrm{C})$ & 4273 & 7468 & 11732 & 77 \\
\hline $\mathrm{H}(9 \mathrm{~A})$ & -464 & 4515 & 2088 & 90 \\
\hline $\mathrm{H}(9 \mathrm{~B})$ & -1244 & 5021 & 2531 & 90 \\
\hline $\mathrm{H}(9 \mathrm{C})$ & -36 & 4642 & 3652 & 90 \\
\hline $\mathrm{H}(10 \mathrm{~A})$ & -223 & 5481 & 689 & 91 \\
\hline $\mathrm{H}(10 \mathrm{~B})$ & 390 & 6149 & 1468 & 91 \\
\hline $\mathrm{H}(10 \mathrm{C})$ & -994 & 5926 & 1254 & 91 \\
\hline $\mathrm{H}(11 \mathrm{~A})$ & 1573 & 4830 & 2180 & 81 \\
\hline $\mathrm{H}(11 \mathrm{~B})$ & 2122 & 4874 & 3780 & 81 \\
\hline $\mathrm{H}(11 \mathrm{C})$ & 2282 & 5483 & 2961 & 81 \\
\hline $\mathrm{H}(14 \mathrm{~A})$ & -1060 & 6275 & 3019 & 44 \\
\hline $\mathrm{H}(16 \mathrm{~A})$ & -1127 & 7620 & 4077 & 50 \\
\hline $\mathrm{H}(16 \mathrm{~B})$ & -2085 & 7012 & 3625 & 50 \\
\hline $\mathrm{H}(17 \mathrm{~A})$ & -980 & 7567 & 6242 & 52 \\
\hline $\mathrm{H}(18 \mathrm{~A})$ & -1485 & 6442 & 6255 & 85 \\
\hline $\mathrm{H}(18 \mathrm{~B})$ & -2942 & 6597 & 5230 & 85 \\
\hline $\mathrm{H}(18 \mathrm{C})$ & -2323 & 6898 & 6718 & 85 \\
\hline $\mathrm{H}(19 \mathrm{~A})$ & -2487 & 8282 & 4609 & 96 \\
\hline $\mathrm{H}(19 \mathrm{~B})$ & -2952 & 8051 & 5686 & 96 \\
\hline $\mathrm{H}(19 \mathrm{C})$ & -3558 & 7726 & 4219 & 96 \\
\hline
\end{tabular}

Table 5. Torsion angles [deg] for 02388 .

$\mathrm{O}(2)-\mathrm{Cu}(1)-\mathrm{O}(1)-\mathrm{C}(13)$

$-3.5(5)$

$\mathrm{C}(2)-\mathrm{Cu}(1)-\mathrm{O}(1)-\mathrm{C}(13)$

$177.5(5)$ 


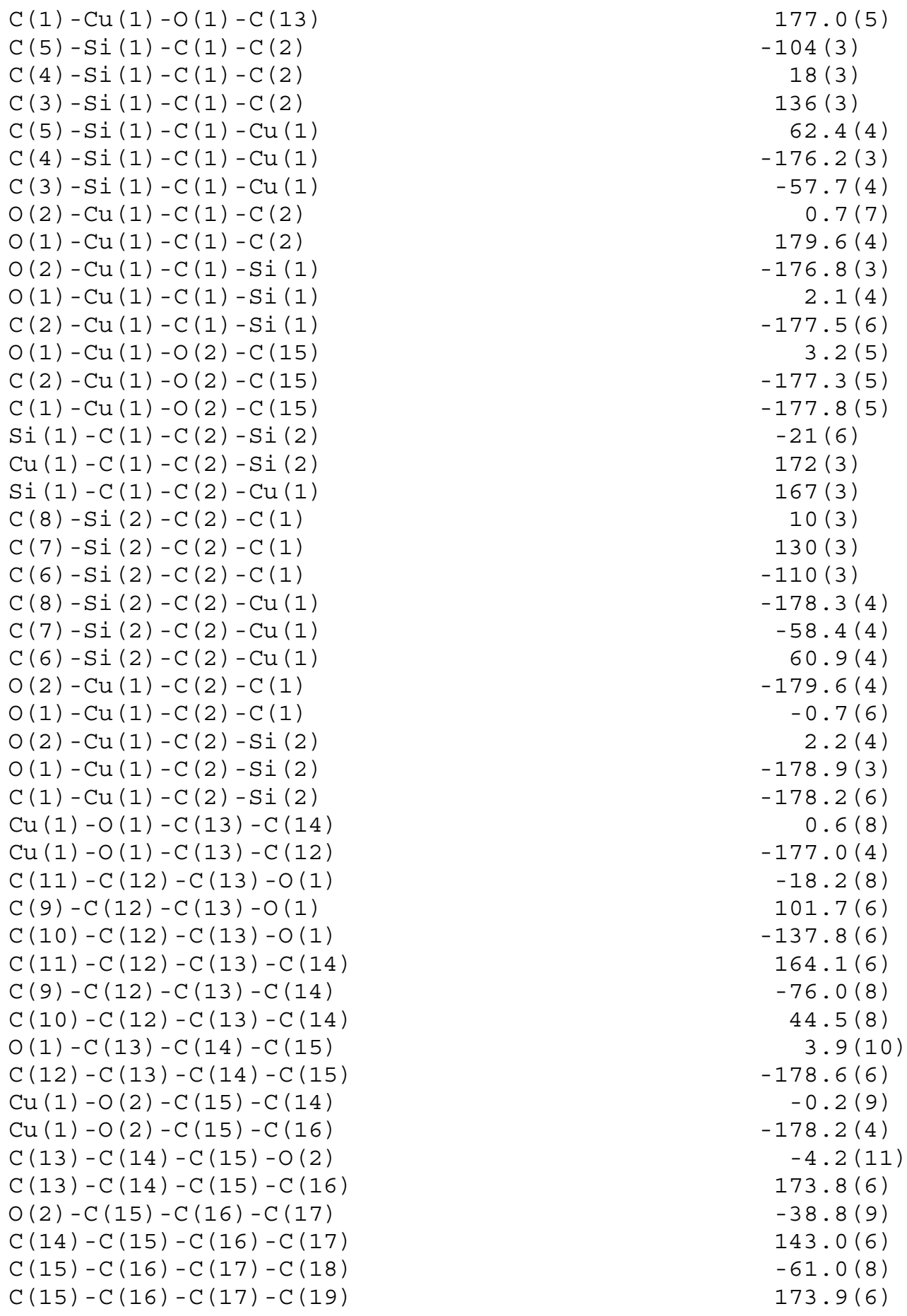




\section{$\underline{\text { X-RAY CRYSTAL STRUCTURE ANALYSIS Compound } 4}$}

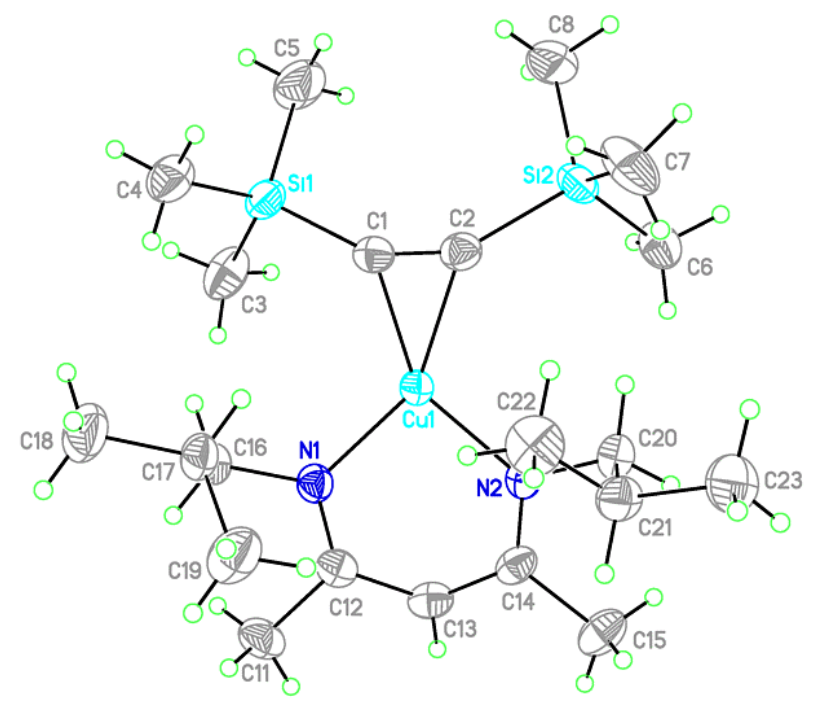

CRYSTAL DATA: C21 H43 Cu N2 Si2, from pentane, cololorless, irregular block, $\sim 0.300 \times 0.300 \times 0.100 \mathrm{~mm}$, triclinic , P-1 , a = 11.502(2) $\mathrm{A}, \mathrm{b}=17.021(3) \AA, \mathrm{c}=$ 6.9570(14) $\AA$, alpha $=96.20(3)^{\circ}$, beta $=103.00(3)^{\circ}$, gamma $=89.58(3)^{\circ}, \mathrm{Vol}=$ 1319.2(5) $\AA^{3}, \mathrm{Z}=2, \mathrm{~T}=-100 .{ }^{\circ} \mathrm{C}$, Formula weight $=443.29$, Density $=1.116 \mathrm{~g} / \mathrm{cm}^{3}$, $\mu(\mathrm{Mo})=0.93 \mathrm{~mm}^{-1}$

DATA COLLECTION: Rigaku RU300, R-AXIS image plate area detector, MoKalpha radiation, filament size $=12.0 \times 2.0 \mathrm{~mm}$, anode power $=55 \mathrm{kV} \times 220 \mathrm{~mA}$, crystal to plate distance $=85.0 \mathrm{~mm}, 105 \mu$ pixel raster, number of frames $=45$, oscillation range $=4.0^{\circ}$, exposure time $=8.0 \mathrm{~min} /$ frame, box sum integration, hkl $\mathrm{min} / \mathrm{max}=(-13,13,-19,18,-7$, 7 ), data input to shelx $=7087$, unique data $=3717$, two-theta range $=3.64$ to $48.20^{\circ}$, completeness to two-theta $48.20=88.90 \%, \mathrm{R}(\mathrm{int}-\mathrm{xl})=0.0515$, Because test datasets have showed Isigma's from Raxis software to be unrealistically small, A value of 5.0 has been added to all Isigma's.

SOLUTION AND REFINEMENT: Structure solved using XS(Shelxtl), refined using shelxtl software package, refinement by full-matrix least squares on $\mathrm{F}^{2}$, scattering factors from Int. Tab. Vol C Tables 4.2.6.8 and 6.1.1.4, number of data $=3717$, number of restraints $=0$, number of parameters $=248$, data/parameter ratio $=14.99$, goodness-of-fit on $\mathrm{F}^{2}=0.95, \mathrm{R}$ indices[ $\left.\mathrm{I}>4 \operatorname{sigma}(\mathrm{I})\right] \mathrm{R} 1=0.0360, \mathrm{wR} 2=0.0878, \mathrm{R}$ indices(all data) $\mathrm{R} 1$ $=0.0448, \mathrm{wR} 2=0.0906$, max difference peak and hole $=0.343$ and $-0.477 \mathrm{e} / \AA^{3}$, All of the hydrogen atoms have been idealized as riding hydrogens. The rotation of the methyl groups are refined.

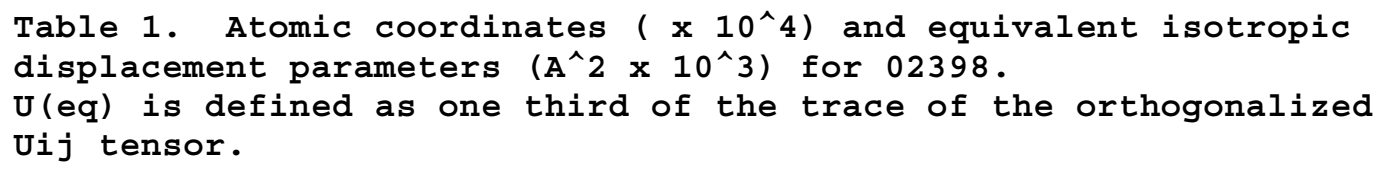




\begin{tabular}{|c|c|c|c|c|}
\hline & $\mathbf{x}$ & $y$ & z & $\mathrm{U}(\mathrm{eq})$ \\
\hline $\mathrm{Cu}(1)$ & $7924(1)$ & $2777(1)$ & $1703(1)$ & $25(1)$ \\
\hline Si (1) & $7534(1)$ & $872(1)$ & $-1042(1)$ & $35(1)$ \\
\hline $\mathrm{N}(1)$ & $9624(2)$ & $2807(1)$ & $1752(4)$ & $28(1)$ \\
\hline$C(1)$ & $7120(3)$ & $1776(2)$ & $400(4)$ & $31(1)$ \\
\hline Si (2) & $4772(1)$ & $2444(1)$ & $934(1)$ & $36(1)$ \\
\hline $\mathrm{N}(2)$ & $7795(2)$ & $3872(1)$ & $2720(3)$ & $26(1)$ \\
\hline$C(2)$ & $6362(3)$ & $2218(2)$ & $937(5)$ & $32(1)$ \\
\hline$C(3)$ & $8439(4)$ & $1166(2)$ & $-2751(6)$ & $52(1)$ \\
\hline$C(4)$ & $8314(3)$ & $188(2)$ & $714(6)$ & $47(1)$ \\
\hline$C(5)$ & $6133(4)$ & $379(2)$ & $-2552(6)$ & $62(1)$ \\
\hline$C(6)$ & $4375(3)$ & $3379(2)$ & $-198(5)$ & $42(1)$ \\
\hline$C(7)$ & $4521(3)$ & $2470(3)$ & $3482(6)$ & $58(1)$ \\
\hline$C(8)$ & $3843(3)$ & $1624(2)$ & $-626(7)$ & $63(1)$ \\
\hline$C(11)$ & $11439(3)$ & $3524(2)$ & $1450(5)$ & $41(1)$ \\
\hline C (12) & $10150(3)$ & $3483(2)$ & $1659(4)$ & $30(1)$ \\
\hline$C(13)$ & $9592(3)$ & $4220(2)$ & $1756(4)$ & $31(1)$ \\
\hline C (14) & $8557(3)$ & $4410(2)$ & $2476(4)$ & $31(1)$ \\
\hline C (15) & $8379(3)$ & $5288(2)$ & $2970(5)$ & $42(1)$ \\
\hline$C(16)$ & $10347(2)$ & $2104(2)$ & $2135(5)$ & $31(1)$ \\
\hline$C(17)$ & $10442(3)$ & $1873(2)$ & $4222(5)$ & $34(1)$ \\
\hline C (18) & $11190(3)$ & $1128(2)$ & $4518(6)$ & $55(1)$ \\
\hline C (19) & $10952(4)$ & $2540(2)$ & $5822(5)$ & $52(1)$ \\
\hline C (20) & $6901(3)$ & $4132(2)$ & $3875(4)$ & $32(1)$ \\
\hline$C(21)$ & $7385(3)$ & $4179(2)$ & $6123(5)$ & $35(1)$ \\
\hline$C(22)$ & $7728(3)$ & $3370(2)$ & $6783(5)$ & $49(1)$ \\
\hline C (23) & $6455(3)$ & $4560(2)$ & $7179(5)$ & $51(1)$ \\
\hline
\end{tabular}

Table 2. Bond lengths [A] and angles [deg] for 02398.

\begin{tabular}{ll}
\hline $\mathrm{Cu}(1)-\mathrm{N}(2)$ & $1.937(2)$ \\
$\mathrm{Cu}(1)-\mathrm{N}(1)$ & $1.949(2)$ \\
$\mathrm{Cu}(1)-\mathrm{C}(1)$ & $1.973(3)$ \\
$\mathrm{Cu}(1)-\mathrm{C}(2)$ & $1.975(3)$ \\
$\mathrm{Si}(1)-\mathrm{C}(3)$ & $1.855(4)$ \\
$\mathrm{Si}(1)-\mathrm{C}(4)$ & $1.858(4)$ \\
$\mathrm{Si}(1)-\mathrm{C}(1)$ & $1.863(3)$ \\
$\mathrm{Si}(1)-\mathrm{C}(5)$ & $1.864(4)$ \\
$\mathrm{N}(1)-\mathrm{C}(12)$ & $1.318(4)$ \\
$\mathrm{N}(1)-\mathrm{C}(16)$ & $1.466(4)$ \\
$\mathrm{C}(1)-\mathrm{C}(2)$ & $1.243(4)$ \\
$\mathrm{Si}(2)-\mathrm{C}(8)$ & $1.855(4)$ \\
$\mathrm{Si}(2)-\mathrm{C}(7)$ & $1.855(4)$ \\
$\mathrm{Si}(2)-\mathrm{C}(6)$ & $1.860(3)$ \\
$\mathrm{Si}(2)-\mathrm{C}(2)$ & $1.865(3)$
\end{tabular}




\begin{tabular}{|c|c|}
\hline$N(2)-C(14)$ & $1.323(4)$ \\
\hline$N(2)-C(20)$ & $1.479(4)$ \\
\hline$C(11)-C(12)$ & $1.525(4)$ \\
\hline$C(12)-C(13)$ & $1.408(4)$ \\
\hline$C(13)-C(14)$ & $1.414(4)$ \\
\hline$C(14)-C(15)$ & $1.519(4)$ \\
\hline$C(16)-C(17)$ & $1.525(4)$ \\
\hline$C(17)-C(19)$ & $1.522(4)$ \\
\hline$C(17)-C(18)$ & $1.533(4)$ \\
\hline$C(20)-C(21)$ & $1.531(4)$ \\
\hline$C(21)-C(22)$ & $1.520(5)$ \\
\hline$C(21)-C(23)$ & $1.532(4)$ \\
\hline $\mathrm{N}(2)-\mathrm{Cu}(1)-\mathrm{N}(1)$ & $97.04(10)$ \\
\hline $\mathrm{N}(2)-\mathrm{Cu}(1)-\mathrm{C}(1)$ & $148.59(11)$ \\
\hline $\mathrm{N}(1)-\mathrm{Cu}(1)-\mathrm{C}(1)$ & $113.49(12)$ \\
\hline $\mathrm{N}(2)-\mathrm{Cu}(1)-\mathrm{C}(2)$ & $112.43(12)$ \\
\hline $\mathrm{N}(1)-\mathrm{Cu}(1)-\mathrm{C}(2)$ & $150.18(12)$ \\
\hline$C(1)-C u(1)-C(2)$ & $36.70(13)$ \\
\hline$C(3)-S i(1)-C(4)$ & $113.42(18)$ \\
\hline$C(3)-S i(1)-C(1)$ & $109.05(15)$ \\
\hline$C(4)-S i(1)-C(1)$ & $109.02(16)$ \\
\hline$C(3)-S i(1)-C(5)$ & $108.5(2)$ \\
\hline$C(4)-S i(1)-C(5)$ & $108.68(18)$ \\
\hline$C(1)-S i(1)-C(5)$ & $108.05(16)$ \\
\hline $\mathrm{C}(12)-\mathrm{N}(1)-\mathrm{C}(16)$ & $119.6(2)$ \\
\hline $\mathrm{C}(12)-\mathrm{N}(1)-\mathrm{Cu}(1)$ & $119.8(2)$ \\
\hline $\mathrm{C}(16)-\mathrm{N}(1)-\mathrm{Cu}(1)$ & $120.22(18)$ \\
\hline$C(2)-C(1)-S i(1)$ & $151.2(2)$ \\
\hline$C(2)-C(1)-C u(1)$ & $71.74(19)$ \\
\hline $\mathrm{Si}(1)-\mathrm{C}(1)-\mathrm{Cu}(1)$ & $136.12(17)$ \\
\hline$C(8)-S i(2)-C(7)$ & $107.7(2)$ \\
\hline$C(8)-\operatorname{Si}(2)-C(6)$ & $108.92(18)$ \\
\hline$C(7)-S i(2)-C(6)$ & $113.38(18)$ \\
\hline$C(8)-S i(2)-C(2)$ & $107.38(17)$ \\
\hline$C(7)-S i(2)-C(2)$ & $110.21(16)$ \\
\hline$C(6)-S i(2)-C(2)$ & $109.09(15)$ \\
\hline$C(14)-N(2)-C(20)$ & $117.5(2)$ \\
\hline $\mathrm{C}(14)-\mathrm{N}(2)-\mathrm{Cu}(1)$ & $120.2(2)$ \\
\hline $\mathrm{C}(20)-\mathrm{N}(2)-\mathrm{Cu}(1)$ & $122.18(19)$ \\
\hline$C(1)-C(2)-S i(2)$ & $150.1(3)$ \\
\hline $\mathrm{C}(1)-\mathrm{C}(2)-\mathrm{Cu}(1)$ & $71.56(19)$ \\
\hline $\mathrm{Si}(2)-\mathrm{C}(2)-\mathrm{Cu}(1)$ & $137.82(18)$ \\
\hline $\mathrm{N}(1)-\mathrm{C}(12)-\mathrm{C}(13)$ & $123.8(3)$ \\
\hline $\mathrm{N}(1)-\mathrm{C}(12)-\mathrm{C}(11)$ & $121.9(3)$ \\
\hline$C(13)-C(12)-C(11)$ & $114.3(3)$ \\
\hline$C(12)-C(13)-C(14)$ & $127.5(3)$ \\
\hline$N(2)-C(14)-C(13)$ & $123.3(3)$ \\
\hline$N(2)-C(14)-C(15)$ & $121.7(3)$ \\
\hline$C(13)-C(14)-C(15)$ & $115.0(3)$ \\
\hline$N(1)-C(16)-C(17)$ & $112.7(2)$ \\
\hline$C(19)-C(17)-C(16)$ & $112.4(3)$ \\
\hline$C(19)-C(17)-C(18)$ & $110.6(3)$ \\
\hline$C(16)-C(17)-C(18)$ & $110.3(3)$ \\
\hline$N(2)-C(20)-C(21)$ & $113.4(2)$ \\
\hline$C(22)-C(21)-C(20)$ & $111.5(3)$ \\
\hline$C(22)-C(21)-C(23)$ & $111.2(3)$ \\
\hline
\end{tabular}


Table 3. Anisotropic displacement parameters (A^2 x 10^3) for 02398 . The anisotropic displacement factor exponent takes the form: $-2 \mathrm{pi}^{\wedge} 2\left[\mathrm{~h}^{\wedge} 2 \mathrm{a}^{\wedge} 2 \mathrm{U} 11+\ldots+2 \mathrm{~h} k \mathrm{a} * \mathrm{~b} * \mathrm{U} 12\right]$

\begin{tabular}{|c|c|c|c|c|c|c|}
\hline & U11 & U2 2 & U33 & $\mathrm{U} 23$ & U13 & U12 \\
\hline $\mathrm{Cu}(1)$ & $23(1)$ & $23(1)$ & $28(1)$ & $4(1)$ & $6(1)$ & $2(1)$ \\
\hline $\mathrm{Si}(1)$ & $40(1)$ & $25(1)$ & $38(1)$ & $0(1)$ & $6(1)$ & $1(1)$ \\
\hline $\mathrm{N}(1)$ & $27(1)$ & $28(1)$ & $30(1)$ & $6(1)$ & $7(1)$ & $2(1)$ \\
\hline$C(1)$ & $30(2)$ & $31(2)$ & $31(2)$ & $4(1)$ & $4(1)$ & $-3(1)$ \\
\hline $\mathrm{Si}(2)$ & $23(1)$ & $40(1)$ & $46(1)$ & $11(1)$ & $7(1)$ & $0(1)$ \\
\hline $\mathrm{N}(2)$ & $27(1)$ & $26(1)$ & $23(1)$ & $5(1)$ & $4(1)$ & $4(1)$ \\
\hline$C(2)$ & $33(2)$ & $29(2)$ & $34(2)$ & $7(1)$ & $5(1)$ & $-2(1)$ \\
\hline$C(3)$ & $75(3)$ & $38(2)$ & $48(2)$ & $2(2)$ & $22(2)$ & $9(2)$ \\
\hline$C(4)$ & $53(2)$ & $34(2)$ & $54(2)$ & $11(2)$ & $10(2)$ & $1(2)$ \\
\hline$C(5)$ & $63(2)$ & $44(2)$ & $65(3)$ & $-13(2)$ & $-5(2)$ & $-3(2)$ \\
\hline$C(6)$ & $35(2)$ & $49(2)$ & $45(2)$ & $13(2)$ & $11(2)$ & $6(1)$ \\
\hline$C(7)$ & $36(2)$ & $84(3)$ & $64(3)$ & $31(2)$ & $20(2)$ & $2(2)$ \\
\hline$C(8)$ & $34(2)$ & $51(2)$ & $95(3)$ & $4(2)$ & $-1(2)$ & $-6(2)$ \\
\hline$C(11)$ & $30(2)$ & $51(2)$ & $45(2)$ & $2(2)$ & $14(1)$ & $-7(1)$ \\
\hline$C(12)$ & $30(2)$ & $35(2)$ & $24(2)$ & $3(1)$ & $4(1)$ & $-2(1)$ \\
\hline$C(13)$ & $38(2)$ & $29(2)$ & $26(2)$ & $10(1)$ & $5(1)$ & $-7(1)$ \\
\hline$C(14)$ & $38(2)$ & $24(2)$ & $26(2)$ & $2(1)$ & $-1(1)$ & $0(1)$ \\
\hline$C(15)$ & $61(2)$ & $26(2)$ & $39(2)$ & $7(1)$ & $7(2)$ & $2(2)$ \\
\hline$C(16)$ & $28(2)$ & $29(2)$ & $37(2)$ & $0(1)$ & $10(1)$ & $5(1)$ \\
\hline$C(17)$ & $35(2)$ & $29(2)$ & $36(2)$ & $7(1)$ & $6(1)$ & $8(1)$ \\
\hline$C(18)$ & $58(2)$ & $39(2)$ & $59(2)$ & $7(2)$ & $-6(2)$ & $15(2)$ \\
\hline C (19) & $66(2)$ & $41(2)$ & $39(2)$ & $0(2)$ & $-3(2)$ & $6(2)$ \\
\hline$C(20)$ & $35(2)$ & $30(2)$ & $30(2)$ & $3(1)$ & $8(1)$ & $7(1)$ \\
\hline$C(21)$ & $39(2)$ & $38(2)$ & $30(2)$ & $-2(1)$ & $12(1)$ & $-2(1)$ \\
\hline$C(22)$ & $56(2)$ & $55(2)$ & $36(2)$ & $14(2)$ & $5(2)$ & $4(2)$ \\
\hline$C(23)$ & $54(2)$ & $60(2)$ & $42(2)$ & $-5(2)$ & $24(2)$ & $0(2)$ \\
\hline
\end{tabular}

Table 4. Hydrogen coordinates $\left(x 0^{\wedge} 4\right)$ and isotropic displacement parameters $\left(A^{\wedge} 2 \times 10^{\wedge} 3\right)$ for 02398 .

\begin{tabular}{lllll}
\hline $\mathbf{x}$ & $\mathbf{y}$ & $\mathrm{z}$ & $\mathrm{U}(\mathrm{eq})$ \\
\hline
\end{tabular}




\begin{tabular}{|c|c|c|c|c|}
\hline $\mathrm{H}(3 \mathrm{~A})$ & 8732 & 702 & -3388 & 78 \\
\hline $\mathrm{H}(3 \mathrm{~B})$ & 9101 & 1493 & -2013 & 78 \\
\hline $\mathrm{H}(3 \mathrm{C})$ & 7953 & 1455 & -3738 & 78 \\
\hline $\mathrm{H}(4 \mathrm{~A})$ & 7798 & 51 & 1540 & 71 \\
\hline $\mathrm{H}(4 \mathrm{~B})$ & 9026 & 441 & 1529 & 71 \\
\hline $\mathrm{H}(4 \mathrm{C})$ & 8521 & -283 & -19 & 71 \\
\hline $\mathrm{H}(5 \mathrm{~A})$ & 5643 & 238 & -1688 & 93 \\
\hline $\mathrm{H}(5 \mathrm{~B})$ & 6325 & -89 & -3310 & 93 \\
\hline $\mathrm{H}(5 \mathrm{C})$ & 5708 & 734 & -3435 & 93 \\
\hline $\mathrm{H}(6 \mathrm{~A})$ & 3578 & 3520 & -109 & 63 \\
\hline $\mathrm{H}(6 \mathrm{~B})$ & 4420 & 3306 & -1567 & 63 \\
\hline $\mathrm{H}(6 \mathrm{C})$ & 4922 & 3793 & 497 & 63 \\
\hline $\mathrm{H}(7 \mathrm{~A})$ & 4912 & 2032 & 4102 & 88 \\
\hline $\mathrm{H}(7 \mathrm{~B})$ & 3680 & 2435 & 3419 & 88 \\
\hline $\mathrm{H}(7 \mathrm{C})$ & 4840 & 2956 & 4243 & 88 \\
\hline $\mathrm{H}(8 \mathrm{~A})$ & 4086 & 1133 & -111 & 95 \\
\hline $\mathrm{H}(8 \mathrm{~B})$ & 3943 & 1604 & -1963 & 95 \\
\hline $\mathrm{H}(8 \mathrm{C})$ & 3020 & 1711 & -610 & 95 \\
\hline $\mathrm{H}(11 \mathrm{~A})$ & 11966 & 3428 & 2679 & 62 \\
\hline $\mathrm{H}(11 \mathrm{~B})$ & 11607 & 4039 & 1126 & 62 \\
\hline $\mathrm{H}(11 \mathrm{C})$ & 11555 & 3132 & 414 & 62 \\
\hline $\mathrm{H}(13 \mathrm{~A})$ & 9944 & 4630 & 1292 & 37 \\
\hline $\mathrm{H}(15 \mathrm{~A})$ & 8498 & 5408 & 4381 & 64 \\
\hline $\mathrm{H}(15 \mathrm{~B})$ & 7583 & 5424 & 2341 & 64 \\
\hline $\mathrm{H}(15 \mathrm{C})$ & 8943 & 5585 & 2498 & 64 \\
\hline $\mathrm{H}(16 \mathrm{~A})$ & 11142 & 2204 & 1951 & 38 \\
\hline $\mathrm{H}(16 \mathrm{~B})$ & 9997 & 1666 & 1175 & 38 \\
\hline $\mathrm{H}(17 \mathrm{~A})$ & 9636 & 1749 & 4355 & 40 \\
\hline $\mathrm{H}(18 \mathrm{~A})$ & 10857 & 714 & 3496 & 83 \\
\hline $\mathrm{H}(18 \mathrm{~B})$ & 11186 & 962 & 5792 & 83 \\
\hline $\mathrm{H}(18 \mathrm{C})$ & 11995 & 1241 & 4450 & 83 \\
\hline $\mathrm{H}(19 \mathrm{~A})$ & 10464 & 2997 & 5633 & 77 \\
\hline $\mathrm{H}(19 \mathrm{~B})$ & 11750 & 2668 & 5738 & 77 \\
\hline $\mathrm{H}(19 \mathrm{C})$ & 10966 & 2376 & 7104 & 77 \\
\hline $\mathrm{H}(20 \mathrm{~A})$ & 6225 & 3767 & 3500 & 38 \\
\hline $\mathrm{H}(2 \mathrm{OB})$ & 6618 & 4648 & 3533 & 38 \\
\hline $\mathrm{H}(21 \mathrm{~A})$ & 8102 & 4519 & 6473 & 42 \\
\hline $\mathrm{H}(22 \mathrm{~A})$ & 8299 & 3140 & 6080 & 74 \\
\hline $\mathrm{H}(22 \mathrm{~B})$ & 7029 & 3034 & 6502 & 74 \\
\hline $\mathrm{H}(22 \mathrm{C})$ & 8072 & 3423 & 8183 & 74 \\
\hline $\mathrm{H}(23 \mathrm{~A})$ & 6750 & 4580 & 8589 & 76 \\
\hline $\mathrm{H}(23 \mathrm{~B})$ & 5728 & 4253 & 6789 & 76 \\
\hline $\mathrm{H}(23 \mathrm{C})$ & 6305 & 5087 & 6821 & 76 \\
\hline
\end{tabular}

Table 5. Torsion angles [deg] for 02398 .

$-24.7(2)$

$147.7(2)$

$146.6(3)$

$147.8(2)$

$-39.7(2)$

$-40.9(4)$ 


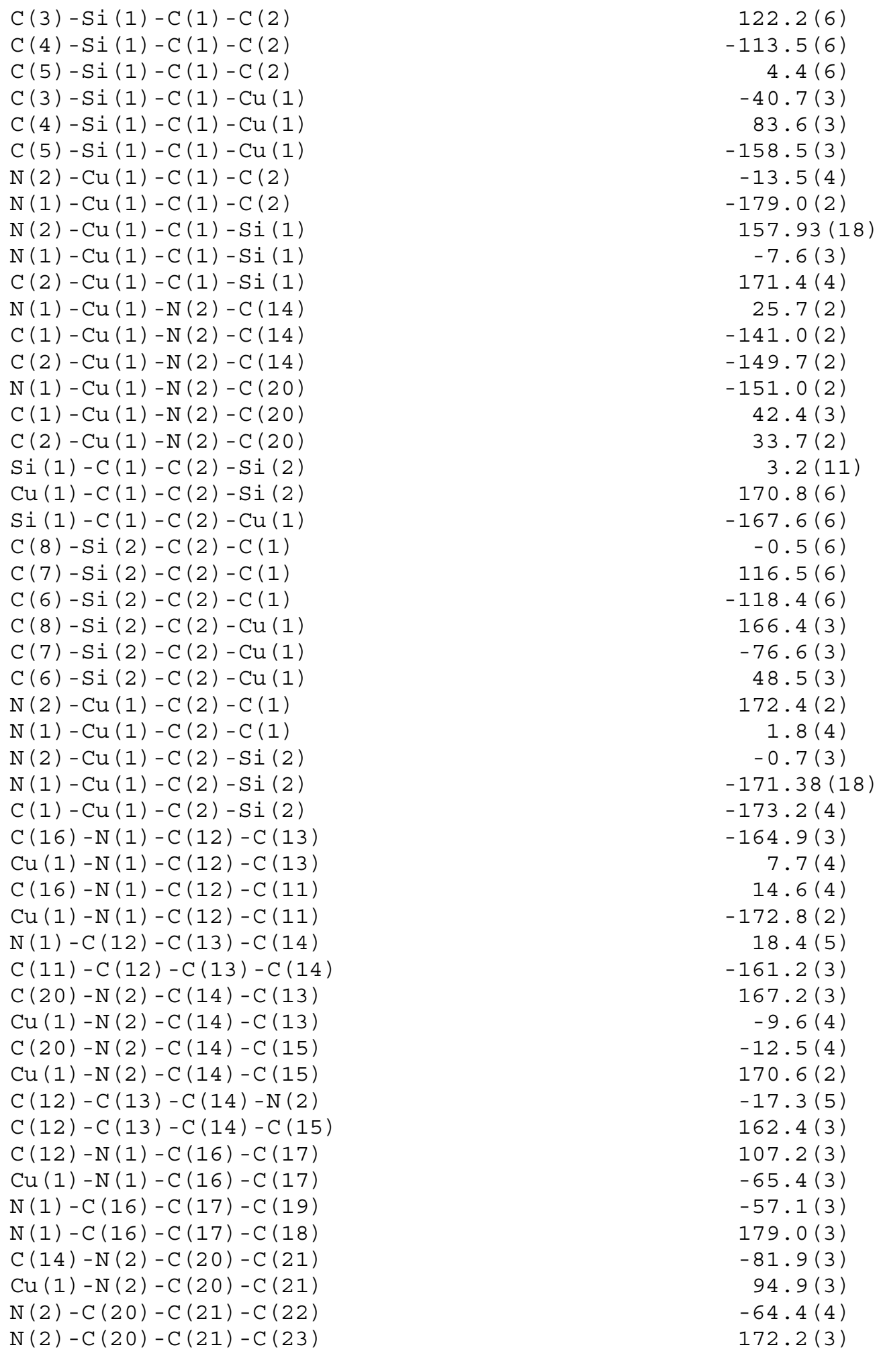

